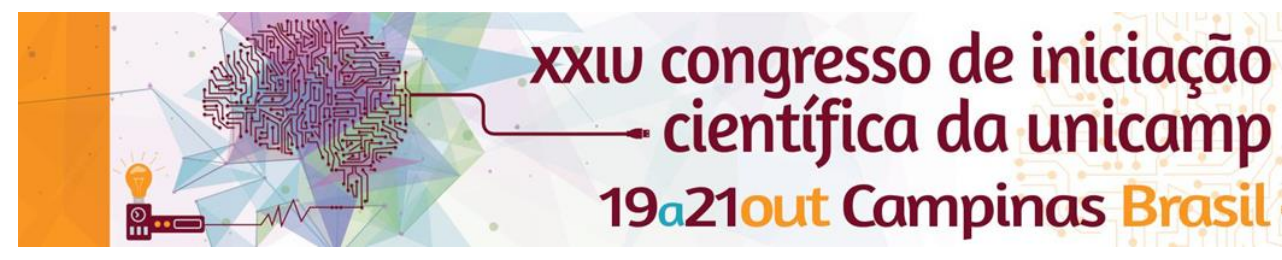

\title{
Electrodeposited nickel nanowires for magnetic-field effect transistor (MagFET)
}

\author{
M. V. Puydinger dos Santos ${ }^{1,2}$, M. Velo ${ }^{1}$, R. D. Domingos ${ }^{1}$, J. Bettini ${ }^{3}$, J. A. Diniz ${ }^{2}$, F. Béron ${ }^{1}$, and K. R. Pirota ${ }^{1}$ \\ 1 - Institute of Physics "Gleb Wataghin", University of Campinas, 13083-859 Campinas, SP, Brazil. \\ 2 - School of Electrical and Computer Engineering and Center for Semiconductor Components, University of \\ Campinas, 13083-970 Campinas, SP, Brazil. \\ 4 - Brazilian Nanotechnology National Laboratory (LNNano), 13083-100 Campinas, SP, Brazil. \\ puyding@ifi.unicamp.br
}

\begin{abstract}
The growing interest in magnetic nanowires (NW) is connected to possibility of employing them for advanced applications in wide technological fields, such as data storage and biotechnology. In addition, NW can be used as sensor devices for several applications, since they present high sensitivity to the environment. One of the major challenges when dealing with transport measurements in NW is to trap them between electrodes, which allows electrical characterization and therefore fabrication of nanowire-based devices. This can be used for investigation of electrical transport properties of individual NW and fabrication of NW-based devices, such as sensors and field effect transistors. Especially for ferromagnetic NW, one can use the present method for fabrication of magnetic field-effect transistors (MagFET).
\end{abstract}

\section{Key words:}

nickel nanowires; nanowire-based devices; magnetoresistance, Focused lon Beam (FIB).

\section{Introduction}

Therefore, in this work, properly isolated NiNW, with length of around $4 \mu \mathrm{m}$ and $35 \mathrm{~nm}$ of diameter, obtained by electrodeposition. Optimized electrodes geometry and DEP electrical parameters [13] were taken for NiNW deposition. In addition, electrical characterizations of the NW and of the contact resistance between the NW and electrode were performed by current versus voltage curves. Significant reduction of contact resistance was achieved by ion-beam assisted deposition of Pt cap layers on the NW extremities. The main objective of this work is to study Ni nanowires (NiNW) for the future MagFET applications. This device works based on anisotropic magnetoresistance (AMR), a quantummechanical effect based on spin-orbit coupling, which allows current modulation through the NW by an external magnetic field [16].

\section{Results and Discussion}

The electric resistivity of the NiNW, $\rho$, was measured as a function of temperature, $T$, using a standard four-probe technique in a Physical Property Measurement System (PPMS), in the range of $2-300 \mathrm{~K}$ (Fig. 7), showing metallic behavior, as expected. The residual resistance is $\rho_{0}=27 \Omega . \mathrm{cm}$, which is in good agreement with similar dimensions NiNW [4,18]. The relative ratio of resistivity $\rho(300 \mathrm{~K}) / \rho(4.2 \mathrm{~K})=2$ is much smaller than the value of 47 for the bulk [4]. Since the NW dimensions and grain-size are similar to the mean free path of $\mathrm{Ni}(\sim 10 \mathrm{~nm})$ the increase in resistivity can be attributed mainly to the grain-boundary scattering $[16,17]$.

The variation of $\rho$ as a function of the magnetic field, $H$, was measured at $300 \mathrm{~K}$ for one isolated nanowire with the current flowing perpendicular to the applied field. DOI: 10.19146/pibic-2016-50753
Maximum resistivity value, decreases down to around $1 \%$ as the magnetic field increases, as expected for NiNW. This behavior is attributed to the quantum-mechanical effect of spin-orbit coupling for magnetic fields below 10 kOe. MR $\times \mathrm{H}$ for temperatures of 300,100 and $2 \mathrm{~K}$. Changes in MR with $T$ are closely related to changes in magnetic anisotropy due to the thermal expansion coefficients mismatch between the $\mathrm{SiO}_{2}\left(\alpha_{\mathrm{SiO} 2}=0.65 \times 10^{-6}\right.$ $\left.\mathrm{K}^{-1}\right)$ substrate and the NiNW $\left(\mathrm{\alpha}_{\mathrm{Ni}}=13 \times 10^{-6} \mathrm{~K}^{-1}\right)$. Where $K_{\mathrm{Ni}}$ $=25 \times 10^{10} \mathrm{~N} \cdot \mathrm{m}^{-2}$ is the elastic constant for bulk Ni. For $\Delta T$ $=300 \mathrm{~K}-2 \mathrm{~K}=298 \mathrm{~K}$, the induced stress in a NiNW is approximately $0.92 \mathrm{GN} . \mathrm{m}^{-2}$. Such a tensile stress on the NiNW, which is thermally dependent, can alter its magnetoelastic anisotropy, thus changing the preferred magnetization direction from longitudinal to transversal (parallel to $H$ ). This leads to reduction in MR signal for NiNW.

\section{Conclusions}

NiNW present ferromagnetic properties, which allow their low current levels to be controlled through magnetic fields, like a MagFET device. These magnetotransport properties can be thought as a promising alternative to the traditional Si-based MOSFET devices.

\section{Acknowledgement}

The authors would like to thank CCS/UNICAMP and LMBT/IFGW/UNICAMP staff for device processing and characterization. The work was supported by the Brazilian funding agencies FAPESP, CNPq and CAPES. 